\title{
Development of the Russian version of the Work Rehabilitation Questionnaire. Preliminary evaluation of the social and vocational status of patients with traumatic spinal cord injury
}

\author{
Elena Vasilchenko, Georgy Zoloyev, and Karine Karapetian \\ Federal State Budgetary Scientific and Practical Centre for Medical and Social Evaluation and \\ Rehabilitation of Disabled Persons in Novokuznetsk, Russian Federation
}

\begin{abstract}
Introduction: spinal cord injury is a damaging event that affects person's wellbeing and represents a significant societal problem. Traumatic spinal cord injury (TSCI) occurs mostly among young adults and causes severe impairments in daily living activities and functioning. The Work Rehabilitation Questionnaire (WORQ) based on the International Classification of Functioning, Disability and Health was developed recently as an instrument to evaluate functioning in vocational rehabilitation. The evidence of work activities and vocational status of disabled persons in Russia remains almost unstudied, Therefore, the need for Russian version of WORQ has emerged to investigate the social and vocational status of TSCI-patients. Objectives: cross-cultural adaptation of WORQ into Russian; preliminary evaluation of the social and vocational status of patients with TSCI. Material and methods: Russian WORQ (self-reported version) was used to investigate the social and vocational status of the patients with TSCI. Results: more than $80 \%$ of participants are not vocationally active; Conclusions: the most severe degree of impairment is not associated with lower rates of employment; female patients with TSCI are more likely to have an active working status.
\end{abstract}

Key words: spinal cord injury, permanent disability, The Work Rehabilitation Questionnaire (WORQ), social and vocational status.

\section{Introduction}

Spinal cord injury (SCI) can cause severe impairments in daily living activities and functioning. SCI is one of the leading causes of disability, and it represents a significant societal problem specifically including its increasing incidence and the high cost associated with the treatment of the patients $[1,2]$.

Despite advances made in rehabilitation medicine, which brought an increase in life expectancy and a reduction in mortality rates, SCI remains one of the most devastating health injuries often producing permanent disability. According to limited availability of data on epidemiological studies, the incidence of spinal trauma in Russian industrial cities is 5-6 per $100000[3,4]$. Although there are no accurate and up-to-date formal country-level statistical data on the average age of traumatic spinal cord injury (TSCI) patients, different separate 
studies in this field suggest comparable evidence according to which about $80 \%$ of patients with TSCI are of working age [5, 6]. Being a very disabling condition TSCI affects quality of life and vocational ties, and without an appropriate rehabilitation it makes it extremely hard for person to work and build a career. The importance of this matter is supported by different study reviews providing evidence that there is a relationship between unemployment and suicide [7-10], and longer duration of unemployment is related to a greater risk of suicide and suicide attempt [11].

Given that disabled persons with TSCI are mostly young adults, it is reasonable to suggest that for them unemployment is a vital issue. Nevertheless, despite the consequence of the problem the evidence on work activities and vocational status of this category of disabled persons in Russia remains almost unstudied. Therefore, the need to investigate the social and vocational status of patients with TSCI seems apparent. To accomplish this, it is crucial to choose an appropriate assessment tool. Since it is based on The International Classification of Functioning, Disability and Health (ICF) by the World Health Organization and is targeted to assess and evaluate functioning in vocational rehabilitation settings we decided to use Work Rehabilitation Questionnaire (WORQ) [12].

\section{Material and methods}

\subsection{Assessment tool}

Work Rehabilitation Questionnaire (WORQ) was developed as a generic instrument and can be applied in different populations of patients. It consists of 2 parts comprising a total of 59 points. Part 1 consists of socio-demographic and work-related questions (17 points). Part 2 consists of series of questions about persons functioning (42 points) [13].

To make it possible to use WORQ in our Centre we needed to make its cross-cultural adaptation from English into Russian. This cross-cultural adaptation was made in accordance with the "3-steps Cross Cultural Adaptation" [14] suggested by the authors of the original WORQ; the process of adaptation included following 3 steps:

1) Translation: Forward translation; Independent translation by two translators ( $\mathrm{T} 1$ and T2), preferably of different background; one can be non-health professional (e.g. linguist, teacher); Must have document comments and rationale; Translation of the instrument instructions, items, and responses.

2) Synthesis: $\mathrm{T} 1$ and $\mathrm{T} 2$ meet to discuss the original instrument and their translations; Document synthesis, issues, and resolutions; producing a new version of the instrument.

3) Expert Committee Review: methodologists, health professionals, language professionals, and translators from Step 1, and 2-3 patients (diverse patients are required: paraplegia/tetraplegia, complete/incomplete lesion); patients need to pre-test translated version; Need all the comments from all versions; Development of the final version, understood by a 12-year-old.

In order to develop the final version, we had to make sure the equivalence between English and Russian versions was retained. So, a linguistic analysis was performed to gain semantic, idiomatic, experiential and conceptual equivalence. An interim report, which included all semantic changes caused by differences between Russian and English cultures in general and languages specifically, was also developed. Eventually, the Russian version of WORQ was approved by the authors of the original version of WORQ [15]. 


\subsection{Participants and settings}

The study was conducted in the Federal State Budgetary Scientific and Practical Centre for Medical and Social Evaluation and Rehabilitation of Disabled Persons in Novokuznetsk, Russia (the Centre).

It was decided to undertake this study in patients with traumatic spinal cord injury because of the increasing number of this population and the severity of medical condition. TSCI-patients is also one of the main populations our Centre provides treatment and rehabilitation for. Hence, we recruited 168 patients with TSCI undergoing treatment course in the Neurosurgery Department of the Centre to investigate their social and vocational status. All participants were older than 18 years. Inclusion criterion was the presence of traumatic spinal cord injury. Exclusion criteria were presence of mental disorders, critical condition, and patient's unwillingness to participate in the study.

\subsection{Data collection}

From February 16, 2016 till September 21, 2016, one hundred and sixty-eight patients have participated in the study. Patients were recruited on their first admission to the Centre regardless of whether the treatment goals were surgical or rehabilitative. During the first two weeks after hospitalization they were asked to fill in the Questionnaire within no longer than three days. Since WORQ is a self-report questionnaire it is usually completed by a patient himself, but some of the participants required a physical assistance, e.g. with writing down the answers as they experienced difficulties with holding a pen because of impaired fine motor skills.

Part 1 of the Questionnaire consists of 17 points and asks socio-demographic and workrelated questions; some of the questions ask patient to describe their current (or last) job, what are (or were) patient's duties, they ask if a change of job is planned, if the patient is undergoing medical or therapeutic treatment, and so on. Part 2 consists of 42 points including series of questions about persons functioning; in this part the patient is asked to rate the extent of their problem in the past week from $0=$ no problem to $10=$ complete problem. To do so the patient is asked to tick the box that best reflects their situation, considering that their answer refers to their ability to function or do an activity without any help from anybody or without any assistive device.

One of the most crucial questions, which is, in essence, a baseline for the comparative analysis, is the question No. 4 "Which best describes your current work status, or if currently not working your last work status?"; the question suggests the following options: 1) Employed, 2) Self-employed, 3) Non-paid work such as volunteer, 4) Student or in training, 5) Homemaker, 6) Retired, 7) Not applicable. With a view to identify the factors influencing patients' vocational activity we divided them into 2 subgroups depending on whether a patient had a job or not. In fact, as can be seen from the analysis, our paper is hinged on this question. On this research stage we only touched on an issue related to some of the socio-demographic questions from Part 1. More detailed analysis, including patients' answers regarding the part about functioning, is needed and to be carried out during the year 2017.

The questionnaires were circulated by a permanent health worker employee of the Centre, who provided orientation on and informed patients about the study and its purpose, and assisted patients when needed.

To describe the clinical characteristics of the sample we decided to collect such additional information about patients as time after injury, level of injury, AIS type, and severity of disability which is determined in accordance with the Russian Federation classification of 
disability. The degree of impairment of the spinal cord conduction was assessed by American Spinal Injury Association Impairment Scale (AIS) [16].

\subsection{Statistical analysis}

Numerical variables are expressed as mean with standard error. For categorical variables, absolute numbers and percentages are shown. The $\chi^{2}$ test was used to check the statistical hypotheses regarding differences in vocational status of patients. If $p$-value was less than 0.05 , the differences were considered as statistically significant.

The data were processed using the standard STATISTICA package (Version 10.0.1011.0, StatSoft Russia, Moscow, Russia).

\subsection{Ethical approval}

Approval for this study was obtained from the Ethics Committee of the Scientific and Practical Centre for Medical and Social Evaluation and Rehabilitation of Disabled Persons in Novokuznetsk. Written informed consent was obtained from all participants.

\section{Results}

\subsection{Sample characteristics}

One hundred and forty-one (84\%) participants were from Siberian Federal District. However, the rest 27 (16\%) patients were from districts all over the Russian Federation: Central, Southern, North-Western, Volga, Ural, and Far Eastern, indicating that the study partially refers to the situation throughout the country.

There were $130(77.3 \%)$ males and $38(22.6 \%)$ females; the average age was $32.2 \pm 0.6$ years; time duration after injury was $5.4 \pm 0.4$ years.

One hundred and fifty-seven (93.4\%) patients had the most severe disability group disability group I, $5(3 \%)$ patients with disability group II, and $2(1.2 \%)$ patients with disability group III. Four patients $(2.4 \%)$ did not have any disability group.

There were $56(33.3 \%)$ patients with AIS A, $51(30.4 \%)$ - AIS B, $40(23.8 \%)$ had AIS C, and $21(12.5 \%)$ patients had AIS D.

\subsection{Analysis}

Of all the examined patients $46(27.4 \%)$ patients were legally married, $23(13.7 \%)$ patients were divorced, $90(53.5 \%)$ patients were never married, 8 (4.7\%) were living with somebody, and $1(0.6 \%)$ patient was widowed.

Investigation of the highest level of education has revealed following information: 2 patients $(1.2 \%)$ have completed primary school (4 years), 24 (14.3\%) - basic general education (9 years), 32 (19\%) patients have completed secondary level of education (11 years), 74 (44\%) patients have completed secondary vocational education (or college), 35 $(20.8 \%)$ patients have completed university education, and $1(0.6 \%)$ patient have completed post-graduate education.

Social link due to family relationships were strong in the majority of patients -165 (97.6\%), and only $1(0.6 \%)$ patient reported poor or absence of family support.

It was found that $22(13 \%)$ patients had a job, $1(0.6 \%)$ was retired, $3(1.8 \%)$ patients were students or in training, and $1(0.6 \%)$ was a homemaker. The overwhelming majority of patients - $141(83.9 \%)$ - were not vocationally active. To ascertain if there were any 
interrelations between person's background and his or her vocational activity, we decided to dig deeper into their social and vocational characteristics and found that:

- among those 22 participants who had a job:

- $17(77.3 \%)$ were self-employed, and $5(22.7 \%)$ were employed.

- $20(90.9 \%)$ patients worked on modified or light duty, $2(9.1 \%)$ patients worked full time.

- $17(77.3 \%)$ patients graduated from either college or university.

- $17(77.3 \%)$ were in the subgroup 1 (had AIS A or AIS B), and 5 (22.7\%) were in the subgroup 2 (had AIS C or AIS D).

- $12(54.5 \%)$ were male participants with average age of $33.2 \pm 1.5$ years, and 10 $(45.5 \%)$ were female participants with average age of $31.3 \pm 2.2$ years.

- $11(50 \%)$ patients were never married, $6(27.3 \%)$ were divorced, $3(13.6 \%)$ were married, and $2(9.1 \%)$ reported living with somebody.

- among those 141 patients who were not vocationally active:

- 41 (29\%) were married, 6 (4.3\%) patients were living with somebody, 76 (54\%) were never married, $17(12 \%)$ were divorced, and $1(0.7 \%)$ patient was widowed.

- $116(82.3 \%)$ were males with average of $31.5 \pm 0.5$ years, $25(17.7 \%)$ were female participants with average of $34 \pm 1.8$ years.

- $90(63.8 \%)$ patients had graduated from either college or university.

- $90(63.8 \%)$ were in the subgroup 1 (had AIS A or AIS B), and 51 (36.2\%) were in the subgroup 2 (had AIS C or AIS D).

Considering that "the presence of AIS A or AIS B neurological deficit markedly increases the risk of more severe impairments in body functions and activities and participation" [17], we suggested that patients with more severe impairments would be less likely to be vocationally active. Hence, while analysing the collected data we divided the sample into 2 subgroups: 1 ) with absent or severely impaired spinal cord conduction (AIS A and AIS B) - 107 (63.7\%) patients; and 2) with partially impaired conduction (AIS C and AIS D) - 61 (36.3\%) patients. In fact, our findings revealed that the most severe degree of impairment was not associated with the lower rates of employment $(p=0.14)$.

In the initial stage of this study we also suggested the higher level of education would be associated with the higher rates of employment. That suggestion did not prove to be correct either $(p=0.27)$.

There is a statistically significant difference between working status for males and females: it was found that female patients with TSCI were more likely to have an active working status $(p=0.006)$.

Civil status did not appear to be statistically significant in terms of social and vocational activity $(p=0.35)$.

Working disabled persons held following positions: 3 PC operators; 2 dispatchers, 1 translator, 1 assistant manager, 1 voice teacher, 2 mechanics, 4 salesmen, and 1 head of an organisation.

Four $(2.4 \%)$ patients reported engagement in vocational training activities such as acquiring knowledge and skills for a job, including school training; 15 (8.9\%) reported they were looking for a (new) job or work; all 22 participants who had a job and 1 patient on training reported they were engaged in activities to secure or maintain their current job; 126 (75\%) were neither engaged in any vocational program, nor looking for a (new) job. 


\section{Discussion}

Using WORQ, we assessed the main components of the social and vocational activities of 168 patients with TSCI. All data collected were analysed to establish the current situation among patients with traumatic spinal cord injury regarding their social and vocational status.

Study results reveal numbing figures: first, employment rates among TSCI-patients are very low (13\%); secondly, majority of the participants $(n=126,75 \%)$ not only were unemployed, but also reported not to be engaged in any activity to change their current state. Given that employment is related to the gender but is not associated with either severity of neurologic deficit or level of education, it is reasonable to claim this population of patients has a great potential to be more socially and vocationally active, to be reintegrated into society, and, consequently, to become more independent. Social and medical examination institutions should turn their attention to this population, investigate it profoundly, and assume measures to provide employment opportunities in a consistent and efficient manner.

Despite the fact that data collection process is complicated by many refusals of patients to participate, we keep collecting new cases as well as do follow-ups at 3,6, and 12-months checkpoints after the first assessment. We believe follow-up data can allow us to observe the dynamics of the situation.

\section{Conclusions}

Most of the vocationally active patients were self-employed and worked on modified or light duty. The most severe degree of impairment isn't associated with the lower rates of employment; female patients with TSCI are more likely to have an active working status. For the purpose of finding out what are, if there are any, the interrelations between patient's functioning and vocational activities further investigation is needed.

\section{References}

[1] G.E. Ivanova, V.V. Krylov, M.B. Cykunov, B.A. Poljaev (eds), Rehabilitation of Patients with Spinal Cord Injury (Mos. b. and cart.-lith, 2010) (in Russian)

[2] M.M. Kosichkin, L.P. Grishina, D.M. Shapiro, Med. Soc. Eval. Rehabil. 1, 9-15 (1999)

[3] A.N. Barinov, E.N. Kondakov, Spine Surg. 4, 15-18 (2010)

[4] I.N. Morozov, S.G. Mlyavych, Med. Anthol. 4, 157-159 (2011)

[5] M.A. Leontyev, O.D. Ovchinnikov, Bull. Kuzb. Sci. Cen. SBRAMS 1, 131-136 (2005)

[6] I.A. Sionova, E.N. Kondakov, Proceedings of the III Congress of Neurosurgeons of Russia (2002)

[7] R.L. Jin, C.P. Shah, T.J. Svoboda, CMAJ 153, 529 (1995)

[8] S. Platt, Soc. Sci. Med. 19, 93-115 (1984)

[9] S.H. Wilson, G.M. Walker, Publ. Health 107, 153-162 (1984)

[10] S. Stack, Suic. Life Threat. Behav. 30, 145-162 (2000)

[11] A. Milner, A. Page, A.D. LaMontagne, PLoS One 8, e51333 (2013)

[12] M.E. Finger, R. Escorpizo, C. Bostan, R. De Bie. J. Occup. Rehabil. 24, 498-510 (2014)

[13] R. Escorpizo, S. Brage, D. Homa, G. Stucki (eds), Handbook of Vocational Rehabilitation and Disability Evaluation (Handb. Health, Work, Disabil, 2015)

[14] J. Epstein, R.H. Osborne, G.R. Elsworth, D.E. Beaton, F. Guillemin, J. Clin. Epidemiol. 68, 360-369 (2015) 
[15] Work Rehabilitation Questionnaire [Electronic resource], Mode of access: http:// myworq.org/questionnaire_ru.php Date of access: 21.12.2016

[16] S.C. Kirshblum, W. Waring, F. Biering-Sorensen, S.P. Burns, M. Johansen, M. Schmidt-Read et al., J. Spinal. Cord Med. 34, 547-554 (2011)

[17] E. Vasilchenko, R. Escorpizo, et al. A descriptive study on the functioning profile of patients with spinal cord injury in a rehabilitation center in Russia (Spinal Cord, 2016) 